\title{
LAPM: a tool for underwater large-area photo-mosaicking
}

\author{
Y. Marcon, H. Sahling, and G. Bohrmann \\ MARUM - Center for Marine Environmental Sciences and Faculty of Geosciences, University of Bremen, Bremen, Germany \\ Correspondence to: Y. Marcon (ymarcon@marum.de)
}

Received: 18 March 2013 - Published in Geosci. Instrum. Method. Data Syst. Discuss.: 27 March 2013

Revised: 27 June 2013 - Accepted: 1 July 2013 - Published: 5 July 2013

\begin{abstract}
This paper presents a new tool for large-area photo-mosaicking (LAPM tool). This tool was developed specifically for the purpose of underwater mosaicking, and it is aimed at providing end-user scientists with an easy and robust way to construct large photo-mosaics from any set of images. It is notably capable of constructing mosaics with an unlimited number of images on any modern computer (minimum $1.30 \mathrm{GHz}, 2 \mathrm{~GB}$ RAM). The mosaicking process can rely on both feature matching and navigation data. This is complemented by an intuitive graphical user interface, which gives the user the ability to select feature matches between any pair of overlapping images. Finally, mosaic files are given geographic attributes that permit direct import into ArcGIS. So far, the LAPM tool has been successfully used to construct geo-referenced photo-mosaics with photo and video material from several scientific cruises. The largest photo-mosaic contained more than 5000 images for a total area of about $105000 \mathrm{~m}^{2}$. This is the first article to present and to provide a finished and functional program to construct large geo-referenced photo-mosaics of the seafloor using feature detection and matching techniques. It also presents concrete examples of photo-mosaics produced with the LAPM tool.
\end{abstract}

\section{Introduction}

Low visibility in the deep sea constrains images of the seafloor to being taken from a very short distance $(<10 \mathrm{~m})$ to the intended target. Such low altitude severely limits the field of view of individual images. Hence, creating a photomosaic to encompass an entire community or selected site requires many images.

Photo-mosaicking consists of aligning and stitching photographs together to form a large composite picture. A sim- ilar technique is widely used in photography for the production of panoramas. However, an interest in using photomosaics to map deep-sea environments is growing among the scientific community, and several works have focused on developing algorithms to reliably build underwater mosaics (Gracias and Santos-Victor, 2001; Eustice et al., 2002; Pizarro and Singh, 2003; Vincent et al., 2003; Allais et al., 2004; Ferrer et al., 2007; Escartín et al., 2008). Indeed, traditional panorama-dedicated programs usually fail to cope with underwater imagery correctly. The deep sea is a very challenging environment, and the reasons for failure are manifold; moving camera, strong lighting heterogeneities, low contrasts, perspective distortions, poor accuracy of camera positioning data, or adverse camera motions due to bottom currents are the various hindrances to the construction of large photo-mosaics with low distortion.

Efforts have been made by several research institutes of physics, robotics or computer vision sciences to develop tools to generate photo-mosaics of the seafloor, and impressive results were achieved (Eustice et al., 2002; Pizarro and Singh, 2003; Ferrer et al., 2007; Escartín et al., 2008). However, applying those techniques often requires strong mathematics, physics and programming skills, which the end-user of deep-sea mosaics does not necessarily possess. Indeed, published works about mosaicking techniques are generally aimed at improving known techniques rather than on developing a robust end-user product for deep-sea scientists. For instance, current mosaicking works now concentrate on developing 3-dimensional imaging and mosaicking techniques (Nicosevici et al., 2006, 2009; Brandou et al., 2007; Pizarro et al., 2009), but no freely available tool exists for end-users to routinely produce large 2-dimensional areal mosaics based on robust feature-matching techniques. Escartín et al. (2008) proposed a MosaicViewer software to assemble mosaics, 
which relied on geo-referencing information for each image, but it did not include any feature-matching capability.

Nevertheless, marine scientists require mosaicking techniques for the study of deep-sea ecosystems. In marine research, mosaics are often used to conduct spatial analyses in extreme environments such as hydrothermal vents and marine cold seeps (Grehan and Juniper, 1996; Jerosch et al., 2006, 2007a, b; Olu-Le Roy et al., 2007; Podowski et al., 2009; Lessard-Pilon et al., 2010a, b; Barreyre et al., 2012; Mittelstaedt et al., 2012; Rogers et al., 2012; Marsh et al., 2012; Marcon et al., 2013a, b). Such analyses are varied and include for instance descriptions of faunal habitats and distribution, quantifications of areas or population densities as well as qualitative and geo-statistical spatial correlations. Most of these works use different mosaicking techniques, which highlights the lack of standardized mosaicking methods.

The large-area photo-mosaicking tool (LAPM tool) was developed to bring together several functionalities for the first time to meet the needs of deep-sea scientists. The main strength of this tool lies in its ability to combine image geo-referencing data and robust feature-matching techniques, and to generate photo-mosaics of any size and on virtually any modern computer. Furthermore, completed mosaic files are given geographic attributes and can be directly imported into a geographic information system (GIS). Finally, the mosaicking process can be fully controlled through a graphical user interface (GUI), which makes it easy for end users to use without requiring detailed mathematics, physics and programming knowledge. The LAPM tool is available for download in its current version together with a step-by-step user manual at http://doi.pangaea.de/10.1594/ PANGAEA.808960.

\section{Computer requirements}

The LAPM tool was developed in Matlab and compiled to an executable, which can be run without Matlab. It is able to run on Windows, MacOS and Linux operating systems, and only requires the Matlab R2010a runtime (provided with LAPM tool) to be installed. No particular computer requirements other than those of the Matlab runtime are necessary. Currently the program does not implement any multicore parallelization, but this will be implemented in future versions in order to reduce computing times. Nevertheless, it worked flawlessly on a 64 bit Windows 7 platform running on a 1.30 GHz Dual-Core Processor with 4 GB RAM, which is the minimum configuration the LAPM tool was tested with.

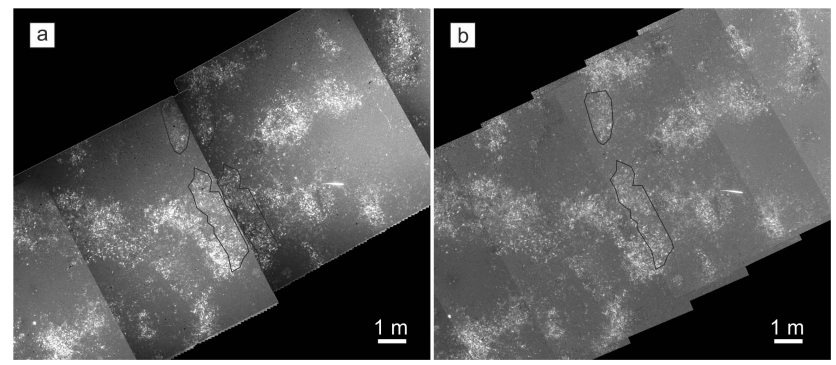

Fig. 1. Two photo-mosaics of a same area, constructed with different methods. The photo-mosaics represent clam aggregates on soft sediments. (a) Navigation-based photo-mosaic; mismatches occur between consecutive images. (b) Feature-based photo-mosaic; better fit between consecutive images; however, more images are required to cover the same area.

\section{Mosaicking with the LAPM tool}

\subsection{Background}

During the photo-mosaicking process, images are aligned together onto a single 2-dimensional plane of the mosaic. The projection and alignment of an image onto the 2-D plane of the mosaic is called image registration (Zitová and Flusser, 2003). For underwater applications, images can be registered in different ways. First by geo-referencing each individual photograph based on navigation (easting, northing, and altitude) and attitude (pitch, roll, and yaw) data of a remotely operated vehicle (ROV) or autonomous underwater vehicle (AUV). The method is fast and requires few computing resources but the quality of the mosaic suffers from any inaccuracies in the navigation data (Fig. 1a). Another method consists of using common features in consecutive images to infer the motion between two images. Such a feature-based method requires each image to overlap with the next and has higher computing requirements, but it generally yields more accurate results (Fig. 1b). However, it may not be able to register images if the overlap area is too small, if the scene is featureless, or if the relief is too sharp.

A robust underwater mosaicking tool should rely on feature tracking methods to ensure best registration accuracy. However, it should also be able to use navigation data in areas where feature tracking fails or is not possible. With the LAPM tool (Fig. 2), mosaics can be constructed in three different ways, depending on the user requirements and on the data available: with feature tracking and navigation data, with feature tracking only, or with navigation data only.

\subsection{Feature tracking}

If used, feature tracking is the first step of the mosaicking process. It consists of detecting and matching features within each consecutive pair of the image sequence. The feature tracking is based on the VLFeat toolbox (Vedaldi and 


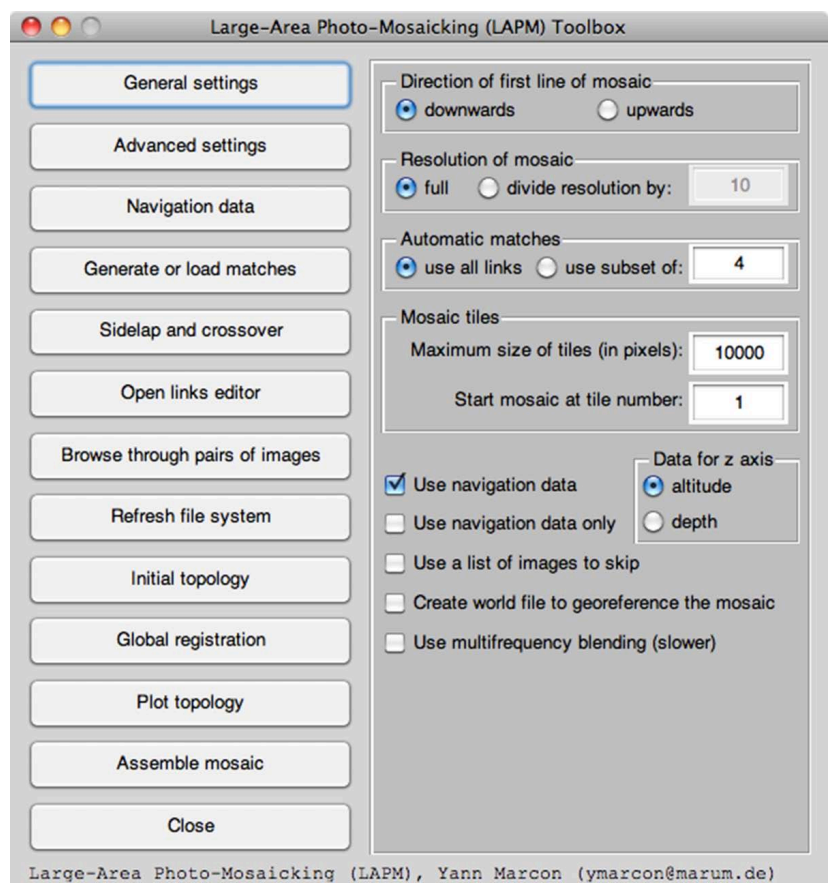

Fig. 2. Graphical user interface (GUI) of the large-area photomosaicking (LAPM) tool; the column of buttons is designed to guide the user through the mosaicking process. Several menus allow the end user fully control the tool.

Fulkerson, 2008, 2010), which includes an open-source implementation of the scale invariant feature transform (SIFT) method (Lowe, 1999, 2004). With this method, a set of features is computed for every image and tracked onto the next consecutive image in order to compute the transform matrix, or homography, that explains the motion. In addition, an outlier rejection (Pizarro and Singh, 2003) is performed in order to remove erroneous matches (outliers) from the set of positive matches (inliers), which ensures that the best possible homography is computed.

In some cases, the feature tracking may fail to compute matches. This happens generally if the overlap between the images is insufficient, if the scene is featureless, or if the relief causes perspective distortions. Such failure can be overcome by user intervention, i.e., by creating a few links between the unmatched images (Fig. 3). Alternatively, navigation data can be used to estimate the motion between unmatched images.

\subsection{Initial topology and crossover}

In mosaicking terms, the topology is the estimation of the position and orientation of every image onto the 2-D plane of the mosaic (Gracias and Negahdaripour, 2005). Computing the topology is the next step of the mosaicking process and a pre-requisite for the actual construction of the mosaic. The "initial topology" step of the LAPM tool consists of estimat-

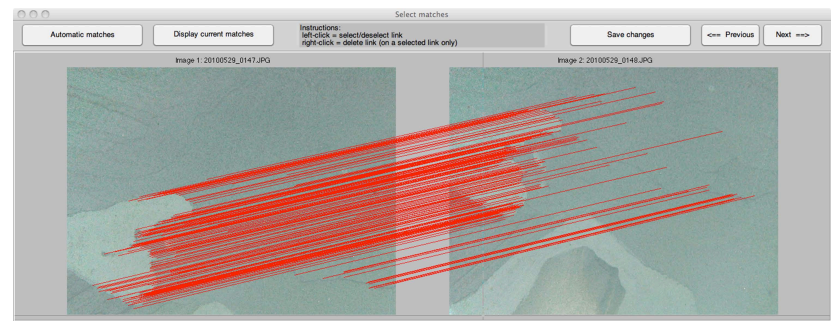

Fig. 3. The interface of the match selector allows the end user the ability to browse through each pair of images and visualize the computed matches; erroneous matches can be removed and new matches can be manually created.

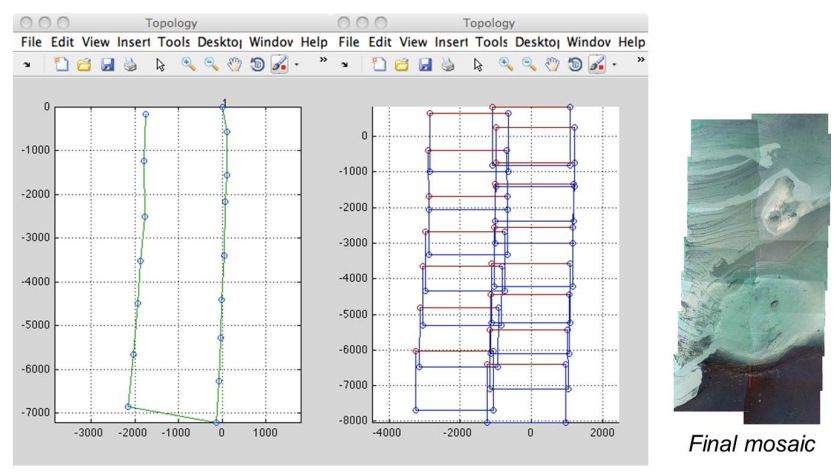

Fig. 4. Illustration of the initial topology. Left: estimation of the center position of each image. Middle: estimation of the projection of the image frames onto the 2-D plane of the mosaic. Right: corresponding mosaic.

ing the position of each image onto the mosaic plane from the homographies (Fig. 4). In this step, the image registration is done successively from the first image to the last in the order of the image sequence.

In such context, minor registration errors tend to add up from one pair to the next and to lead to a larger global error. The use of overlaps between non-consecutive images (Fig. 5) can help limiting the error by providing additional positioning constraints (Fig. 6), also known as crossover points (Fleischer et al., 1996, 1997; Fleischer and Rock, 1998). Crossover points can also be used to compensate for inaccuracies in the navigation data. However, to take advantage of crossover points, overlaps between non-consecutive images must be planned at the image acquisition stage, by preparing an adequate trajectory (Fig. 5).

During mosaicking, crossover points are used in the computation of the initial topology after the pairwise registration of all images has been estimated. Starting again from the first image, whenever a crossover point is found, the registration parameters of all images within the loop are recalculated backward until the beginning of the loop or until the previous crossover point. The principle of this technique is comparable to the smoother-follower technique described by Fleischer et al. (1996). 

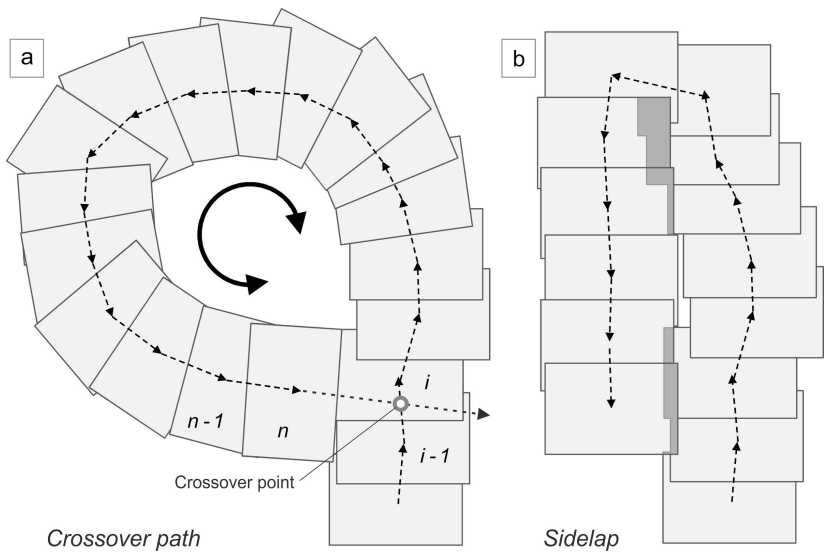

Fig. 5. Examples of crossover trajectories. (a) A crossover path is a trajectory, in which the camera crosses its own trajectory and surveys a same point several times; adapted from Fleischer et al. (1996). (b) Side-overlap, or sidelap, occurs when parallel lines of mosaic overlap (dark grey areas); in this case, the camera trajectory does not necessarily cross itself.
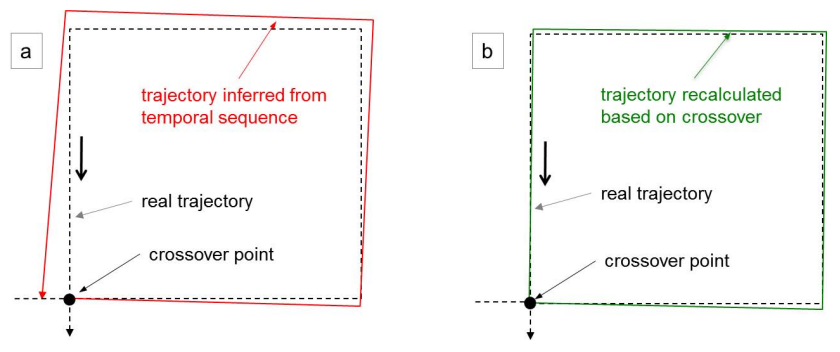

Fig. 6. Illustration of the contribution of crossover points in limiting registration errors. (a) Small positioning errors add up along the trajectory and grow unbounded. (b) By forcing the trajectory to cross itself at a given point, the position of each point within the loop can be recalculated backward; the registration error of each image is reduced.

Concretely, implementing crossover points during the mosaicking process consists of computing or manually adding additional matches between pairs of adjacent (but nonconsecutive) images. Crossover points have been used in previous mosaicking efforts, and in some cases in a fully automatized and iterative fashion (Pizarro and Singh, 2003). In contrast, the LAPM tool gives the user full control over crossover matches. It includes graphical interfaces to display all existing matches and to identify potential crossover points before starting the construction of the final mosaic files. The use of crossover points can significantly improve the quality of the topology and of the final mosaic (Fig. 7).

\subsection{Global registration}

The global registration consists of computing the optimal transformation matrix for each image in order to obtain the smallest possible global error at the scale of the mosaic. This
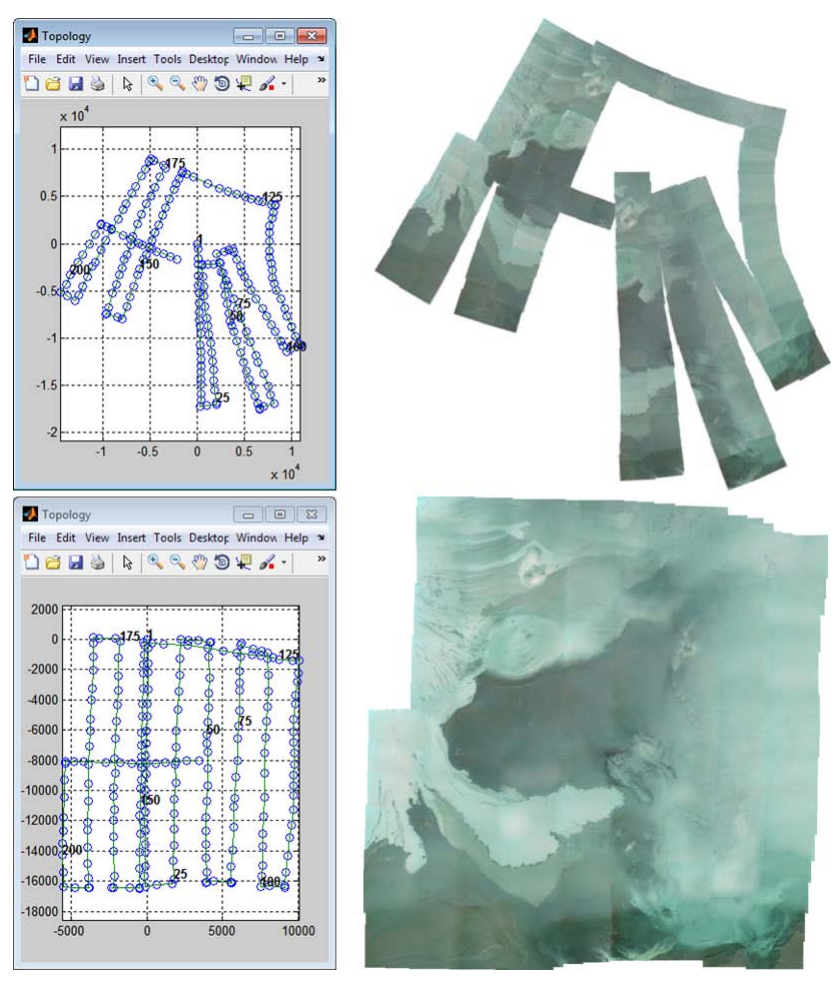

Fig. 7. Illustration of the benefits of using crossover points. Top: pairwise registration errors add up and lead to a huge global error. In this example, the right line of the mosaic corresponds to a featureless area, and the pairwise image registration is impacted by large errors. Bottom: the use of crossover points allows constraining the error. The final mosaic is consistent. Each mosaic line is approximately $20 \mathrm{~m}$ long.

operation, also known as bundle adjustment (Pizarro et al., 2004), is done by minimizing a cost function, which simultaneously takes into account all matches from every matched pair of images.

The LAPM tool uses the cost function presented by Pizarro and Singh (2003) to compute homographies. It is solved in one iteration by linear least squares and presents the advantage of computing the global minimum. The global registration solves for affine homographies; hence, it results in a finer estimation than the initial topology.

\subsection{Mosaic construction}

After all images have been registered, they are merged together to build the actual mosaic. Several methods exist to optimize the rendering of the overlaps, which can be divided in two categories: clipping and blending methods (Burt and Adelson, 1983; Marks et al., 1994; Eustice et al., 2002; Pizarro and Singh, 2003; Ferrer et al., 2007; Lirman et al., 2007; Gracias et al., 2009). Clipping methods consist of taking into account the pixels from one image only out of all overlapping images; they are usually fast but clear seams are visible at the intersection between overlapping images. 

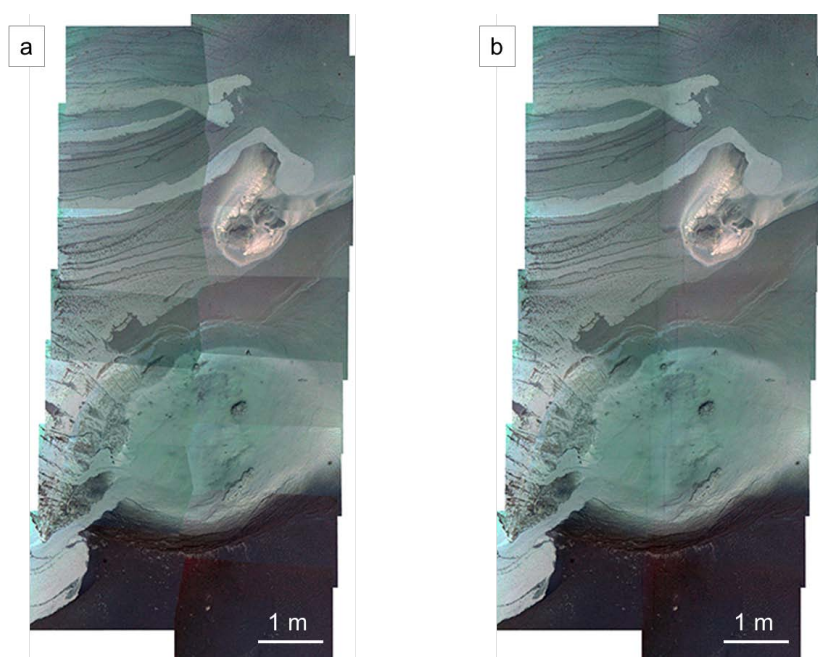

Fig. 8. Illustration of the two rendering options of the LAPM tool. (a) Clipping method: clear seams mark the transition between images. (b) Blending method: seams are barely visible but computing times are longer. The photo-mosaics represent mud pools and mud flows at the Helgoland mud volcano (Black Sea).

Conversely, blending methods merge pixels from the overlapping images; depending on the blending type, such technique can better render the mosaic, although at a higher computing cost.

Two rendering methods are available with the LAPM tool: a clipping method, in which the pixels that are closest to the center of their image are chosen (Lirman et al., 2007), and a blending method, known as multi-resolution pyramidal blending (Eustice et al., 2002; Pizarro and Singh, 2003). The latter method is significantly slower but produces a quasiseamless mosaic (Fig. 8).

\section{LAPM tool: functionalities}

The LAPM tool was developed specifically for the purpose of underwater mosaicking, and its main functions were designed to meet the needs of an end-user scientist interested in building visual maps of the seafloor. Therefore, it has a graphical user interface (GUI) to ensure ease of use without requiring prior knowledge of the techniques involved (Fig. 2). Other main requirements included the abilities (1) to assemble large high-resolution image datasets on any regular computer, (2) to produce geo-referenced mosaics, (3) to give the user the possibility to edit feature matches, and (4) to produce mosaics with lower resolutions.

\subsection{Assembling large image datasets}

Due to a limited field of view in the deep sea, mosaicking areas larger than a few tens of square meters commonly requires hundreds to thousands of images. Most panorama-dedicated programs, such as Autopano Pro, Hugin

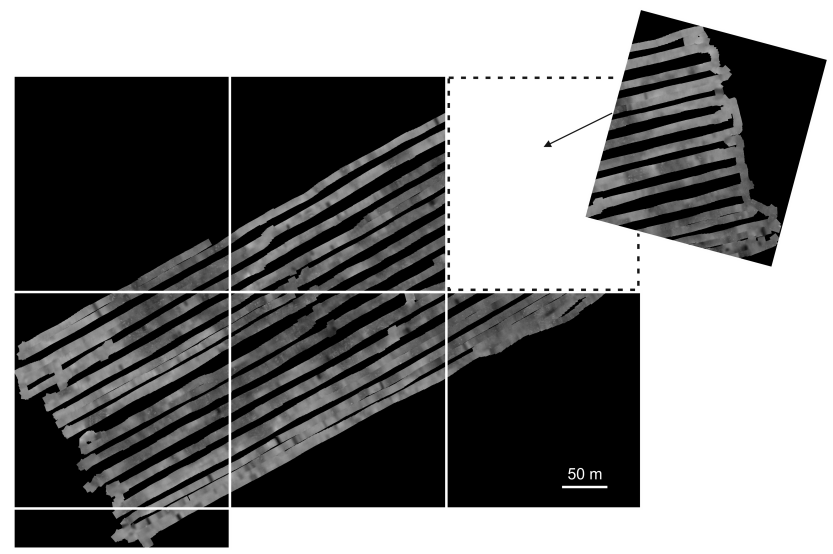

Fig. 9. Illustration of the mosaic tiles as produced by the LAPM tool; in this example, each square tile contains $10000 \times 10000$ pixels and covers about $50000 \mathrm{~m}^{2}$. Individual tiles are constructed separately in order not to exceed the computing resources; tiles are geo-referenced, hence, loading them into a GIS allows displaying the photo-mosaic entirely. The photo-mosaic contains 5421 images and covers a $105000 \mathrm{~m}^{2}$-large area of the Regab pockmark (Lower Congo basin).

or Photoshop ${ }^{\circledR}$, were usually not designed to cope with such large datasets, and computer resources are often exceeded. For instance, a photo-mosaic created using a set of several thousand high-resolution photographs can easily reach a few hundred million pixels. Most computers and operating systems cannot cope with such large files.

The LAPM tool can generate photo-mosaics of any size and on any modern computer with at least 2GB RAM. To achieve such capability, large mosaics are automatically tiled in several square image files (Fig. 9), which are then constructed successively and independently. The tiling is applied during the construction of the mosaic files only and, hence, it does not affect the feature matching and registration steps. The size of individual tiles can be defined by the user and is limited by the computing resources available. Therefore, lower computing capabilities result in smaller tiles. However, because the number of tiles is unlimited, photo-mosaics of any size can be constructed.

For instance, computers equipped with $4 \mathrm{~GB}$ and $64 \mathrm{~GB}$ RAM could build blended mosaic tiles with up to $100 \times 10^{6}$ pixels $(10000 \times 10000)$ and $1.6 \times 10^{9}$ pixels $(40000 \times 40000)$, respectively. Even larger tile sizes can be constructed if the blending option is not used.

\subsection{Geo-referencing the mosaic}

If the navigation data is used, photo-mosaics can be georeferenced. The geo-referencing data is written in a world file, which allows mosaics to be imported directly into a geographic information system (GIS) such as ArcGIS. A world file is created for every tile of the mosaic. The global photomosaic can be visualized entirely by loading each tile into 
GIS (Fig. 9). Therefore, spatial analyses can be performed directly on the entire mosaic, instead of separately on each individual tile.

A world file can also be produced when full navigation data is not available. In such case, the geo-referencing information is estimated from the position and orientation of the first image of the mosaic, as well as from the average field of view of one image, which is given by the user. Therefore, such method only gives a crude approximation of the true geographical data; its accuracy depends strongly on the topography of the surveyed area and on the motion of the camera.

\subsection{Editing feature matches}

Although robust, feature tracking methods are not foolproof and they sometimes fail to detect matches between overlapping images. This is generally the case if the overlap is too small $(<25 \%)$, the mapped area featureless or the perspective distortions too strong.

Therefore, the LAPM tool includes graphical interfaces that provide the possibility to visualize current matches (Fig. 10) and to manually edit them (Fig. 3). If necessary, the user can select and delete individual links, or create new ones between any pair of images. This function is particularly useful when reliable navigation data is not available and that feature tracking fails. It is also very efficient to force the implementation of crossover points even between very lowoverlapping images.

\subsection{Lowering the resolution}

The LAPM tool can produce the final mosaics at several different resolutions. Lowering the resolution reduces computing times significantly. Indeed, the construction of a highresolution photo-mosaic can a very computer-intensive and slow process, and it may sometimes be advantageous to lower the resolution in order to speed up the process. For instance, building large and accurate photo-mosaics can be an iterative process, for which the construction of lowresolution intermediary mosaics may be beneficial; a preliminary mosaic is indeed an efficient way of identifying crossover points in a set of images.

For example, the construction of a $105000 \mathrm{~m}^{2}$ mosaic made of 5421 square images (with 16 bit and $1024 \times 1024$ pixels), on a computer equipped with a $3 \mathrm{GHz} \mathrm{CPU}$, required computing times of about $24 \mathrm{~h}$ without blending and about 4 days with the multi-frequency blending option selected. The final mosaic required six tiles for a total of 5.7 billion pixels. Dividing the resolution of the image by 2 allowed a fully blended mosaic to be constructed within one day.

\section{Results}

The LAPM tool has been used to create maps of several deepsea features. In particular, it produced photo-mosaics with

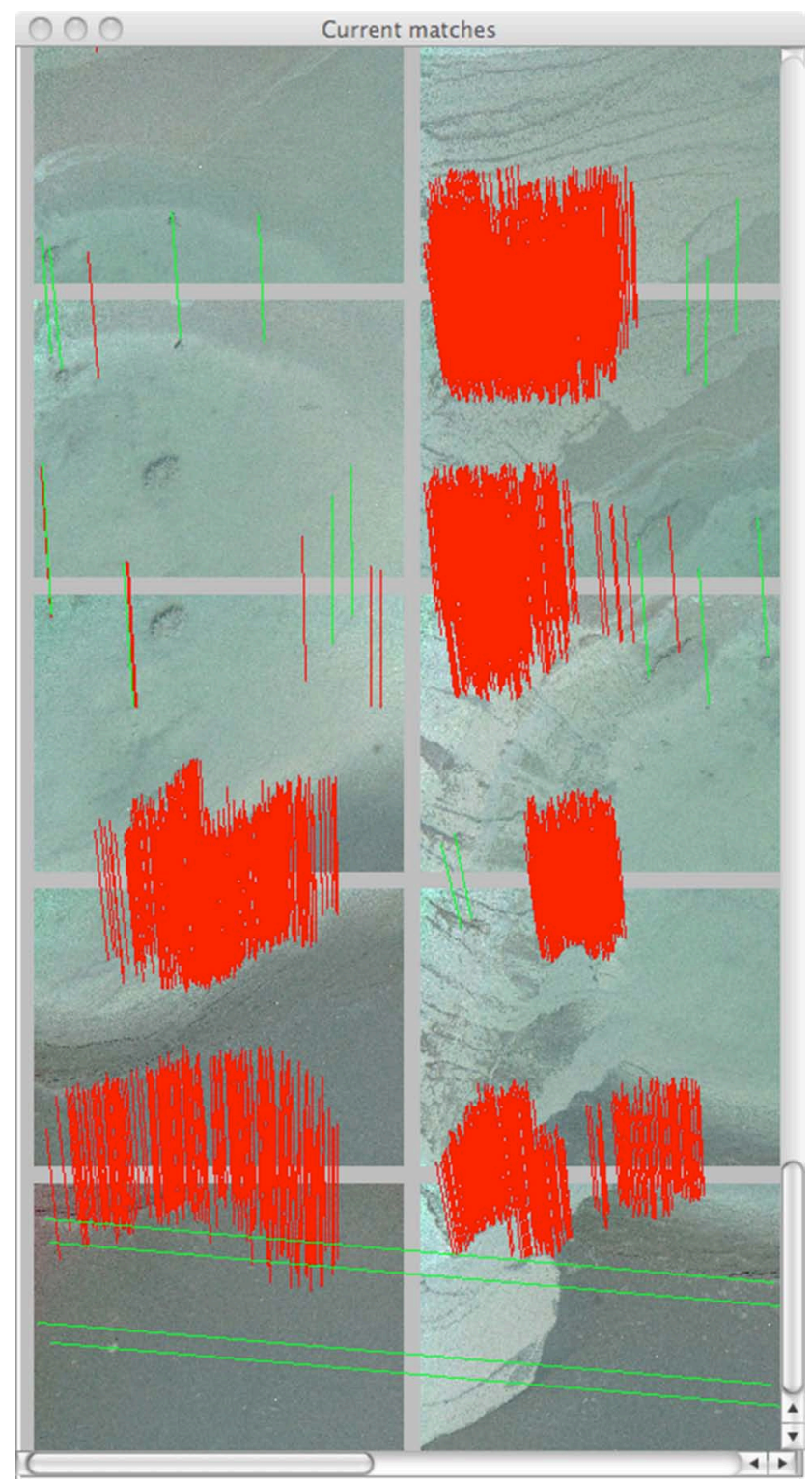

Fig. 10. View of the link editor of the LAPM tool; it gives the possibility to visualize all matches and to identify potential unmatched overlapping images. The red lines represent links that were automatically detected, while the green lines are links which were manually created by the user.

image datasets from different underwater vehicles (ROV and AUV) and from both video- and photo-cameras.

\subsection{Helgoland mud volcano}

The Helgoland mud volcano, in the Black Sea, was visited in 2010 during the MSM15/2 cruise (Bohrmann et al., 2011). High-resolution images of the volcano center were acquired with a Canon Powershot G10 camera mounted on the MARUM ROV Quest 4000. The surveyed areas included both sharp topography and featureless areas, and reliable 


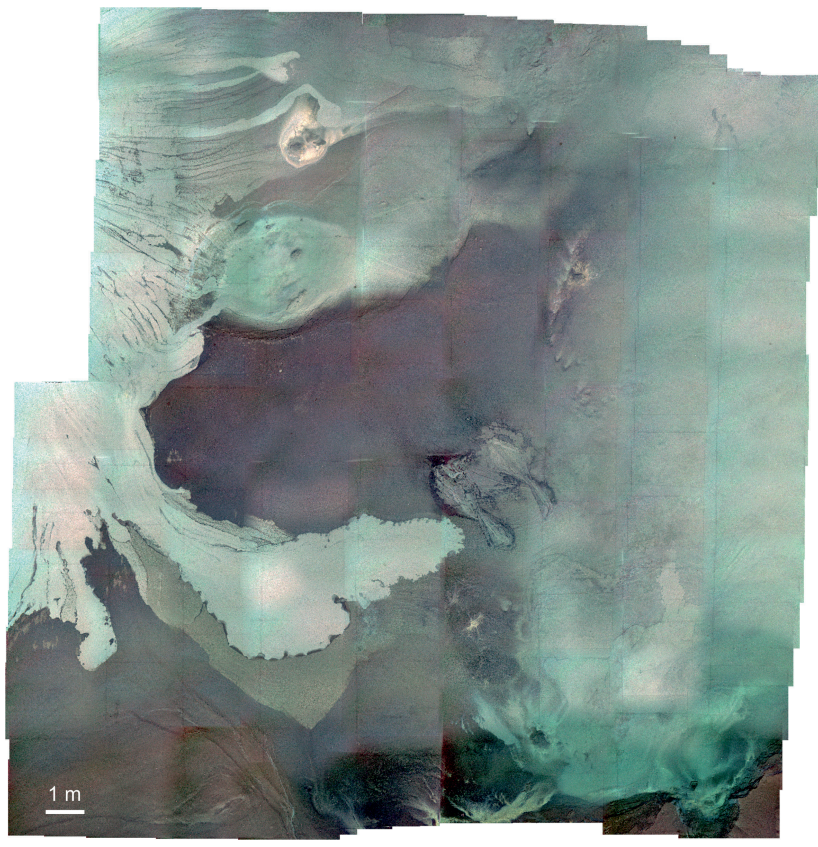

Fig. 11. Photo-mosaic showing mud pool and mud flows at the Helgoland mud volcano; the mosaic was built with 218 images and covers an area of about $400 \mathrm{~m}^{2}$.

ROV-navigation data was not available. Nevertheless, photomosaics could be successfully constructed with the LAPM tool. The results include a $400 \mathrm{~m}^{2}$-large photo-mosaic of the center of the volcano (Fig. 11). The perfect alignment of linear features (mud flows) indicates that the images were accurately registered.

\subsection{Regab pockmark}

The Regab pockmark is a cold seep structure located at $3160 \mathrm{~m}$ water depth in the Congo deep-sea fan. It was intensively studied during the WACS cruise (2011) and large video and photo surveys were conducted with the Ifremer ROV Victor 6000 (Marcon et al., 2013a). Datasets also contain hybrid navigation information from both Doppler Velocity $\log$ (DVL) and ultra-short baseline (USBL) sensors.

Results include notably a $105000 \mathrm{~m}^{2}$-large photo-mosaic of the most active area of the pockmark (Fig. 9). Photos were obtained with the high-sensitivity OTUS camera (Simeoni et al., 2007) from an altitude of $8 \mathrm{~m}$ above the seafloor. Further photo-mosaics could also be constructed from the video materials. To do so, individual frames were extracted at regular interval $(1 \mathrm{~s})$ from the video files. Video images are usually of lower quality than photos from still cameras; however, the high frequency of frames ( $25 \mathrm{fps}$ for PAL cameras) ensured large overlap areas between consecutive images. Therefore, two very high-definition mosaics could be constructed, with areas of $14000 \mathrm{~m}^{2}$ and $5800 \mathrm{~m}^{2}$ (Fig. 12).
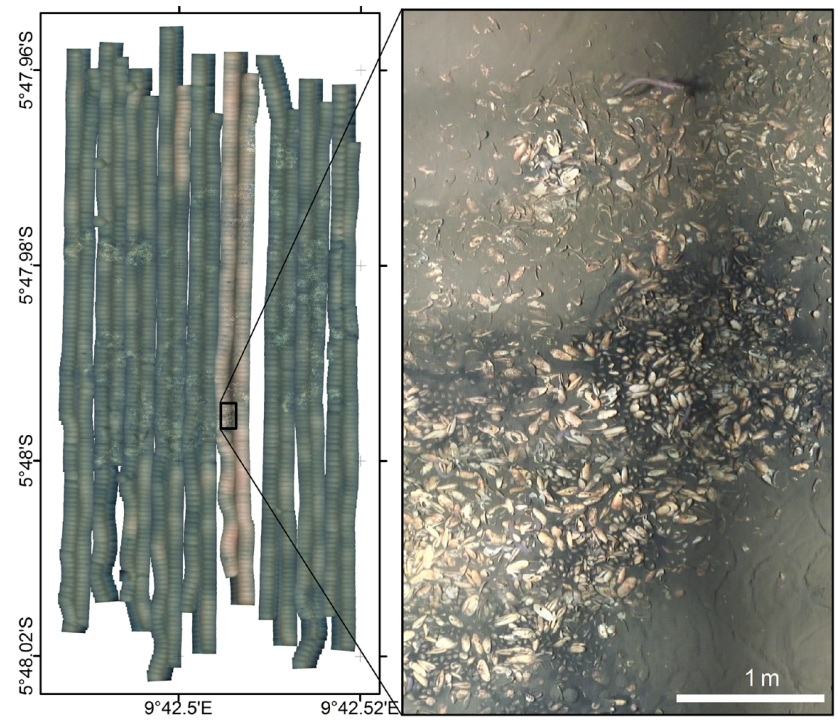

Fig. 12. Geo-referenced photo-mosaic constructed from highdefinition video material of the Regab pockmark (Lower Congo basin); it shows clam aggregations in dark reduced sediments; the photo-mosaic covers a large area of $5800 \mathrm{~m}^{2}$ with a resolution which allows for the ability to distinguish individual living and dead clams.

All mosaics were geo-referenced and could be imported into ArcGIS for spatial analyses.

\subsection{Håkon-Mosby Mud Volcano}

The Håkon-Mosby Mud Volcano (HMMV) is a $1.4 \mathrm{~km}$-wide circular structure located at about $1250 \mathrm{~m}$ water depth in the Barents Sea (Jerosch et al., 2006). The HMMV has been intensively surveyed since its discovery, and high-resolution micro-bathymetry and transects of video-mosaics are already available (Jerosch et al., 2006, 2007b).

Additional photo-mapping surveys were carried out during cruise MSM16/2 (Boetius et al., 2010), with the Sentry AUV from the Woods Hole Oceanic Institute. The largest of these surveys contains more than 5500 photos. The final photomosaic was built using both AUV navigation data and feature tracking, and almost fully covers a $75000 \mathrm{~m}^{2}$-large area of the volcano (Fig. 13). It is geo-referenced and spatial analyses and area calculations can be performed using ArcGIS.

\section{Performance and limitations}

The LAPM tool is based on some of the most robust feature tracking techniques (Lowe, 1999, 2004) currently available as well as some commonly used registration techniques (Pizarro and Singh, 2003). Achieved results show indeed that the LAPM tool can efficiently and accurately register all images into a single mosaic. This is particularly shown by the perfect fits of linear features or the accurate rendering of 


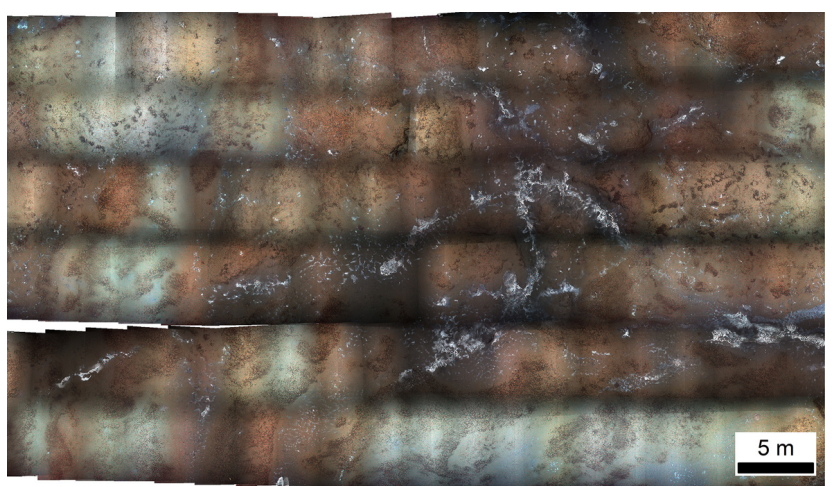

Fig. 13. Excerpt of the photo-mosaic of Håkon-Mosby Mud Volcano; the continuity of the Beggiatoa mats (white patches) and pogonophoran (brown patches) distribution across the mosaic indicates that images were accurately registered. The images were obtained from an altitude of about $5 \mathrm{~m}$.

some faunal aggregations in some of the mosaics presented (Figs. 6, 8, 11-13). For instance, the test dataset shown in Fig. 8 represents downslope mud flows in the Black Sea. It was chosen because it contains many characteristic linear features, which can be used to assess the correctness of the final mosaic. Figure 8 shows that linear features are well reconstructed by the mosaicking process, which indicates that mosaicking errors are low, despite the relief. The Fig. 8 also shows that blurring or degradations of the blended mosaic are also low. This image dataset can be provided upon request for testing purposes.

However, such accuracy can only be achieved in areas where feature matching (either manual or automatic) is possible. If not, the imprecisions of the navigation data are reproduced in the image alignment. Furthermore, since the LAPM tool solves homographies for affine transforms, it intrinsically constructs mosaics with the assumption that the scene is planar. In areas of uneven terrain such as coral reefs, carbonate boulders or hydrothermal vents, relief may cause changes in perspective and in field of view between overlapping images. The program will be able to construct the mosaic anyway, but such changes are likely to cause registration inaccuracies and to impact the quality of final mosaics. Therefore, further analyses based on mosaics of relief areas should be performed knowledgeably. Nevertheless, the same planar scene assumption applies to all homography-based photomosaicking techniques (Gracias and Santos-Victor, 2001; Eustice et al., 2002; Pizarro and Singh, 2003; Ferrer et al., 2007; Escartín et al., 2008), which are also subject to comparable inaccuracies.

In practice studied areas of the seafloor are often characterized by strong relief, which inevitably introduces inaccuracies and misalignments in 2-D mosaics. Therefore, representing such systems with 2-D mosaicking is a challenge, which mainly consists of aligning the images with the minimum global error. However, quantifying such error in mosaick- ing would be necessary to allow marine scientists to better estimate uncertainties in mosaic-based analyses. Generally, such quantification is difficult to achieve since the error is heterogeneously distributed over the mosaic. Nevertheless, a semi-quantitative estimation of the error could be obtained by plotting the final registration residuals for every pair of overlapping images; thus, the user could identify areas of the mosaic where registration errors are the largest and relatively quantify such errors.

The LAPM tool was optimized for efficiency over speed. The structure of the code allows generating mosaics of any sizes without running into memory overflow errors, although at the cost of greater computing times. Furthermore, the LAPM tool does not include multicore parallel computing capability and cannot make full use of modern processors. Nevertheless, not all applications require fullresolution blended mosaics, and processing times can be greatly speeded-up by deactivating the blending option or by reducing the resolution.

Indeed, geometric accuracy is, for marine scientists, by far more important than the quality of the mosaic visual rendering. Although blended seamless mosaics are visually more appealing, they may indeed gum out inaccuracies, such as duplicated features or misalignments. By contrast, unblended mosaics allow the user to visualize areas where errors occur and help to perform analyses knowledgeably.

\section{Conclusions}

The large-area photo-mosaicking LAPM tool was presented in this work. It allows end-users to effectively construct large geo-referenced photo-mosaics without requiring indepth knowledge of the technical aspect of the mosaicking process. The tool can be used with any image data, but its interface and functions were tailored specifically for the purpose of underwater mosaicking. Although based on the current state-of-the-art feature tracking algorithms and on commonly used registration techniques, the LAPM tool does not claim to compete with all other photo-mosaicking techniques. The development of this tool was instead motivated by the current lack of end-user underwater photomosaicking tools freely available to deep-sea scientists interested in seafloor mapping.

It is fully functional and has already been used successfully with photo and video material from several scientific cruises for the production of high quality and high-resolution photo-mosaics. Nevertheless, it will be subject to continuous improvements with notably the ability to correct images for geometric distortions, to solve homographies with projective transformations for finer registration results, to plot registration residuals for better identification of higher-error areas, to use multicore parallel computing, and possibly more robust variants of the feature detection algorithm. 
Acknowledgements. The author would like to thank the Ifremer for its collaboration and contribution during the WACS cruise. Particular thanks to Karine Olu (Ifremer) who provided the images of Regab, and to Michaël Aron for his valuable help and encouragements when getting started with the LAPM tool. Also thanks to Dirk de Beer (Max Plank Institute) and Dana Yoerger (Woods Hole Oceanographic Institute) for providing a large and high quality dataset of the Håkon-Mosby Mud Volcano in order to test the LAPM tool. Finally, thanks to James Collins for proofreading and to the two anonymous reviewers for their comments and suggestions.

This work was supported by SENSEnet, a Marie Curie Initial Training Network (ITN) funded by the European Commission th Framework Programme, Contract No. PITN-GA-2009-237868, as well as by the DFG-Research/Excellence Cluster "The Ocean in the Earth System".

Edited by: M. Syrjäsuo

\section{References}

Allais, A.G., Borgetto, M., Opderbecke, J., Pessel, N., and Rigaud, V.: Seabed video mosaicking with MATISSE: a technical overview and cruise results, in: Proceedings of The Fourteenth International Offshore and Polar Engineering Conference, Toulon, France, 2004.

Barreyre, T., Escartín, J., Garcia, R., Cannat, M., Mittelstaedt, E., and Prados, R.: Structure, temporal evolution, and heat flux estimates from the Lucky Strike deep-sea hydrothermal field derived from seafloor image mosaics, Geochem. Geophys. Geosyst., 13, Q12009, doi:10.1029/2008GC002204, 2012.

Boetius, A., de Beer, D., Felden, J., Viehweger, M., Asendorf, V., Weiz, E., Stiens, R., Eicken, G., Wetzel, G., Harmegnies, F., Dussud, L., Ferré, B., Van Campenhout, J., Albrecht, S., German, C. R., Camilli, R., Billings, A., Kinsey, J. C., Duester, A., Yoerger, D. R., Versteeg, W., Vercruysse, J., and de Rycker, K.: Short Cruise Report - MERIAN MSM16/2 - "LOOME" - Long Term Observatory of Mud Volcano Eruptions (Cruise report), Alfred Wegener Institut für Polar und Meeresforschung, Bremerhaven, 2010.

Bohrmann, G., Blinova, V., Dehning, K., Evtushenko, D., Friese, C., Hiruta, A., Hüttich, D., Ivanov, M., Klapp, S. A., Körber, J. H., Komakhidze, G., Kopiske, E., Lange, K., Mai, H. A., Malakhova, T., Marcon, Y., Meinecke, G., Pape, T., Ratmeyer, V., Rehage, R., Renken, J., Reuter, C., Reuter, M., Römer, M., Sahling, H., Sakvarelidze, E., Wintersteller, P., and Zarrouk, M.: Report and preliminary results of RV MARIA S. MERIAN Cruise MSM 15/2, Istanbul (Turkey) - Piraeus (Greece), 10 May-2 June 2010, Origin and structure of methane, gas hydrates and fluid flows in the Black Sea (Cruise report No. 278), Berichte aus dem Fachbereich Geowissenschaften, Geowissenschaften, Universität Bremen, Bremen, 2011.

Brandou, V., Allais, A. G., Perrier, M., Malis, E., Rives, P., Sarrazin, J., and Sarradin, P. M.: 3D reconstruction of natural underwater scenes using the stereovision system IRIS, in: Proceedings of the MTS/IEEE Oceans 2007 Conference, Vancouver, British Columbia, Canada, 1-6, 2007.

Burt, P. J. and Adelson, E. H.: A multiresolution spline with application to image mosaics, ACM Transactions on Graphics, 2,
217-236, 1983.

Escartín, J., García, R., Delaunoy, O., Ferrer, J., Gracias, N., Elibol, A., Cufi, X., Neumann, L., Fornari, D. J., Humphris, S. E., and Renard, J.: Globally aligned photomosaic of the Lucky Strike hydrothermal vent field (Mid-Atlantic Ridge, $\left.37^{\circ} 18.5^{\prime} \mathrm{N}\right)$ : Release of georeferenced data, mosaic construction, and viewing software, Geochem. Geophys. Geosyst., 9, Q04007, doi:10.1029/2011GC003990, 2008.

Eustice, R., Pizarro, O., Singh, H., and Howland, J.: UWIT: Underwater Image Toolbox for optical image processing and mosaicking in MATLAB, , in: Proceedings of the 2002 International Symposium on Underwater Technology, 2002, Tokyo, Japan, 141-145, 2002.

Ferrer, J., Elibol, A., Delaunoy, O., Gracias, N., and Garcia, R.: Large-area photo-mosaics using global alignment and navigation data, in: Proceedings of the MTS/IEEE Oceans 2007 Conference, Vancouver, British Columbia, Canada, 1-9, 2007.

Fleischer, S. D. and Rock, S. M.: Experimental validation of a realtime vision sensor and navigation system for intelligent underwater vehicles, in: IEEE International Conference on Intelligent Vehicles, Stuttgart, Germany, 1998.

Fleischer, S. D., Wang, H. H., Rock, S. M., and Lee, M. J.: Video mosaicking along arbitrary vehicle paths, in: Proceedings of the 1996 Symposium on Autonomous Underwater Vehicle Technology, 1996. AUV'96, Monterey, CA, 293-299, 1996.

Fleischer, S. D., Rock, S. M., and Burton, R.: Global position determination and vehicle path estimation from a vision sensor for real-time video mosaicking and navigation, in: OCEANS'97. MTS/IEEE Conference Proceedings, Halifax, Nova Scotia, 641647, 1997.

Gracias, N. and Santos-Victor, J.: Underwater mosaicing and trajectory reconstruction using global alignment, in: Proceedings of the MTS/IEEE Oceans 2001 Conference, Honolulu, Hawaii, USA, 7 pp., 2001.

Gracias, N., Mahoor, M., Negahdaripour, S., and Gleason, A.: Fast image blending using watersheds and graph cuts, Image and Vision Computing, 27, 597-607, 2009.

Gracias, X. and Negahdaripour, S.: Underwater mosaic creation using video sequences from different altitudes, in: Proceedings of the MTS/IEEE Oceans 2005 Conference, Washington, DC, 1295-1300, 2005.

Grehan, A. J. and Juniper, S. K.: Clam distribution and subsurface hydrothermal processes at Chowder Hill (Middle Valley), Juan de Fuca Ridge, Mar. Ecol. Prog. Ser., 130, 105-115, 1996.

Jerosch, K., Schlüter, M., and Pesch, R.: Spatial analysis of marine categorical information using indicator kriging applied to georeferenced video mosaics of the deep-sea Håkon Mosby Mud Volcano, Ecol. Inform., 1, 391-406, 2006.

Jerosch, K., Lüdtke, A., Schlüter, M., and Ioannidis, G. T.: Automatic content-based analysis of georeferenced image data: Detection of Beggiatoa mats in seafloor video mosaics from the Håkon Mosby Mud Volcano, Comput. Geosci., 33, 202-218, 2007a.

Jerosch, K., Schlüter, M., Foucher, J. P., Allais, A. G., Klages, M., and Edy, C.: Spatial distribution of mud flows, chemoautotrophic communities, and biogeochemical habitats at Håkon Mosby Mud Volcano, Mar. Geol., 243, 1-17, 2007 b.

Lessard-Pilon, S. A., Podowski, E. L., Cordes, E. E., and Fisher, C. R.: Megafauna community composition associated with Lophelia 
pertusa colonies in the Gulf of Mexico, Deep-Sea Res. Pt. II, 57 , 1882-1890, 2010a.

Lessard-Pilon, S. A., Porter, M. D., Cordes, E. E., MacDonald, I. R., and Fisher, C. R.: Community composition and temporal change at deep Gulf of Mexico cold seeps, Deep-Sea Res. Pt. II, 57, 1891-1903, 2010b.

Lirman, D., Gracias, N., Gintert, B. R., Gleason, A. C., Reid, R. P., Negahdaripour, S., and Kramer, P.: Development and application of a video-mosaic survey technology to document the status of coral reef communities, Environ. Monit. Assess., 125, 59-73, 2007.

Lowe, D. G.: Object recognition from local scale-invariant features, in: The Proceedings of the Seventh IEEE International Conference on Computer Vision, 1999, Presented at the The Proceedings of the Seventh IEEE International Conference on Computer Vision, 1999, Corfu, Greece, 1150-1157, 1999.

Lowe, D. G.: Distinctive image features from scale-invariant keypoints, Int. J. Comput. Vision, 60, 91-110, 2004.

Marcon, Y., Sahling, H., Allais, A. G., Bohrmann, G., and Olu, K.: Distribution and temporal variation of mega-fauna at the Regab pockmark (Northern Congo Fan), based on a comparison of videomosaics and geographic information systems analyses, Mar. Ecol., doi:10.1111/maec.12056, in press, 2013a.

Marcon, Y., Sahling, H., Borowski, C., dos Santos Ferreira, C., Thal, J., and Bohrmann, G.: Megafaunal distribution and assessment of total methane and sulfide consumption by mussel beds at Menez Gwen hydrothermal vent, based on geo-referenced photomosaics, Deep-Sea Res. Pt. I, 75, 93-109, 2013 b.

Marks, R. L., Rock, S. M., and Lee, M. J.: Real-time video mosaicking of the ocean floor, in: Proceedings of the 1994 Symposium on Autonomous Underwater Vehicle Technology, 1994, AUV '94., Brest, France, 21-27, 1994.

Marsh, L., Copley, J. T., Huvenne, V. A. I., Linse, K., Reid, W. D. K., Rogers, A. D., Sweeting, C. J., and Tyler, P. A.: Microdistribution of Faunal Assemblages at Deep-Sea Hydrothermal Vents in the Southern Ocean, PLoS ONE, 7, 19 pp., 2012.

Mittelstaedt, E., Escartín, J., Gracias, N., Olive, J. A., Barreyre, T., Davaille, A., Cannat, M., and Garcia, R.: Quantifying diffuse and discrete venting at the Tour Eiffel vent site, Lucky Strike hydrothermal field, Geochem. Geophys. Geosyst., 13, 18 pp., 2012.

Nicosevici, T., Negahdaripour, S., and Garcia, R.: Monocular-based 3-D seafloor reconstruction and ortho-mosaicing by piecewise planar representation, in: Proceedings of the MTS/IEEE Oceans 2005 Conference, Washington, DC, 1279-1286, 2006.

Nicosevici, T., Gracias, N., Negahdaripour, S., and Garcia, R.: Efficient three-dimensional scene modeling and mosaicing, J. Field Robotics, 26, 759-788, 2009.
Olu-Le Roy, K., Caprais, J. C., Fifis, A., Fabri, M. C., Galéron, J., Budzinsky, H., Le Ménach, K., Khripounoff, A., Ondréas, H., and Sibuet, M.: Cold-seep assemblages on a giant pockmark off West Africa: spatial patterns and environmental control, Mar. Ecol., 28, 115-130, 2007.

Pizarro, O. and Singh, H.: Toward large-area mosaicing for underwater scientific applications, IEEE J. Oceanic Eng., 28, 651-672, 2003.

Pizarro, O., Eustice, R., and Singh, H.: Large area 3D reconstructions from underwater surveys, in: Proceedings of the MTS/IEEE Oceans 2004 Conference, Kobe, Japan, 678-687, 2004.

Pizarro, O., Eustice, R., and Singh, H.: Large area 3D reconstructions from underwater surveys, IEEE J. Oceanic Eng., 34, 150169, 2009.

Podowski, E. L., Moore, T. S., Zelnio, K. A., Luther III, G. W., and Fisher, C. R.: Distribution of diffuse flow megafauna in two sites on the Eastern Lau Spreading Center, Tonga, Deep-Sea Res. Pt. I, 56, 2041-2056, 2009.

Rogers, A. D., Tyler, P. A., Connelly, D. P., Copley, J. T., James, R., Larter, R. D., Linse, K., Mills, R. A., Garabato, A. N., Pancost, R. D., Pearce, D. A., Polunin, N. V. C., German, C.R., Shank, T., Boersch-Supan, P. H., Alker, B. J., Aquilina, A., Bennett, S. A., Clarke, A., Dinley, R. J. J., Graham, A. G. C., Green, D. R. H., Hawkes, J. A., Hepburn, L., Hilario, A., Huvenne, V. A. I., Marsh, L., Ramirez-Llodra, E., Reid, W. D. K., Roterman, C. N., Sweeting, C. J., Thatje, S., and Zwirglmaier, K.: The Discovery of New Deep-Sea Hydrothermal Vent Communities in the Southern Ocean and Implications for Biogeography, PLoS Biol., 10, e1001234, doi:10.1371/journal.pbio.1001234, 2012.

Simeoni, P., Sarrazin, J., Nouze, H., Sarradin, P. M., Ondréas, H., Scalabrin, C., and Sinquin, J. M.: Victor 6000: new high resolution tools for deep sea research, "Module de Mesures en Route," in: Proceedings of the MTS/IEEE Oceans 2007 Conference, IEEE, Vancouver, British Columbia, Canada, 6 pp., 2007.

Vedaldi, A. and Fulkerson, B.: VLFeat: An open and portable library of computer vision algorithms, available at: www.vlfeat. org (last access: 5 July 2013), 2008.

Vedaldi, A. and Fulkerson, B.: VLFeat: An open and portable library of computer vision algorithms, in: Proceedings of the International Conference on Multimedia, Firenze, Italy, 1469-1472, 2010.

Vincent, A. G., Pessel, N., Borgetto, M., Jouffroy, J., Opderbecke, J., and Rigaud, V.: Real-time geo-referenced video mosaicking with the MATISSE system, in: Proceedings of the MTS/IEEE Oceans 2003 Conference, San Diego, USA, 2319-2324, 2003.

Zitová, B. and Flusser, J.: Image registration methods: a survey, Image and Vision Computing, 21, 977-1000, 2003. 